\title{
IMPACT OF THE METAL EVAPORATION RATE IN VACUUM INTERRUPTERS ON VAPOUR EXPANSION AND DEPOSITION
}

\author{
A. E. Geisler*, N. WenZel \\ Siemens AG, Corporate Technology, Research in Energy and Electronics, 91050, Erlangen, Germany \\ * andreas.ag.geisler@siemens.com
}

\begin{abstract}
The emission of contact material into vacuum after switching operation of a vacuum interrupter is crucial for the metallisation of the ceramic surfaces. This work focuses on the simulation of various regimes of metal vapour pressure using an extended version of an existing DSMC code that now allows the visualisation of the interaction types and locations during the vapour expansion. The model was applied to a typical vacuum interrupter geometry at different current levels between $3 \mathrm{~A}$ and $100 \mathrm{kA}$. The simulations show that in the low current case the likelihood for a particle hitting a ceramic surface can be more than a factor of 5 higher than in the high current case. An explanation of this observation will be given by analysing the interaction history of the respective particles.
\end{abstract}

Keywords: vacuum interrupter, DSMC method, vapour deposition, insulator coating, vacuum arc.

\section{Introduction}

Arcs in vacuum emit metal vapour; sometimes this is a desired effect (e.g. in vacuum arc deposition applications), in other cases it can result in an undesired performance degradation of the arcing device (e.g. in vacuum interrupters (VIs)) [1-8]). In terms of computational simulations of this process not only is the high-pressure gradient a numerical challenge, but also the absolute pressure regimes that exist in parallel such as in vacuum interrupters during the switching operation. The Direct Simulated Monte Carlo (DSMC) method [9], however, can be applied for all pressure regimes. In this respect, one important parameter in fluid dynamics is the Knudsen number $K$, defined as the ratio of the mean free path of the gas particles and the typical dimension of the geometry. Even though the DSMC method is less efficient for $K \ll 1$, where the Navier-Stokes equations can be applied, this method can be used for all $K$ as long as the particle interaction time is short compared to the time of the particle flight between two interactions. In our simulations the flight between two collisions is a free flight of neutral copper atoms, but basically the method allows to account for an external force acting on each particle.

This work focuses on the simulation of various regimes of metal vapour pressure using an extended version of an existing DSMC code [10] that now allows the visualization of the interaction types and locations during the vapour expansion.

\section{The DSMC method}

The DSMC method is well described and validated in [11-13]. It is a standard numerical method for rarefied gas dynamics. Rarefied gases are those for which the total volume of the atoms or molecules is small compared to the system volume, i.e. $n V_{\text {atom }} \ll 1$, with

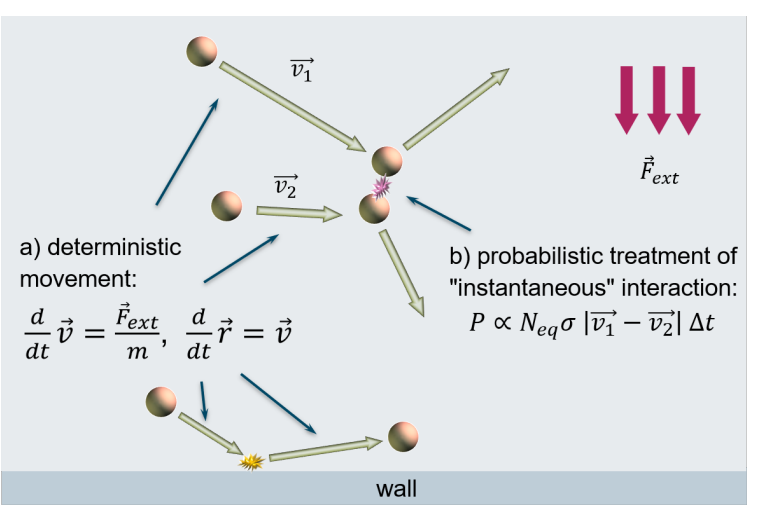

Figure 1. The basic concept of the DSMC method. Decoupling of free flight and collisions during a time step: a) 'conventional' integration of the equation of motion between the interactions taking a possible external force into account, and b) 'dice rolling' for the interactions treated as an instantaneous stochastic process. The likelihood $P$ of an interaction and - in case of an successfull hit - the energy transfer to internal degrees of freedom as well as the post-collisional particle velocities are calculated by random numbers obeying a probability distribution function that corresponds to a selectable interaction model.

$n$ being the atom/molecule density. This is fulfilled even for air at normal conditions. In this case the interaction can be approximated by an instant change of momentum. The basic idea of DSMC is now to introduce a sort of super-particles (DSMC particles), each representing an equivalent number $N_{\text {eq }}$ of real atoms or molecules with an adapted collision crosssection $\sigma_{\mathrm{DSMC}}=N_{\mathrm{eq}} \sigma$, and to divide the processes in a deterministic movement between two collisions and a probabilistic (Monte Carlo driven) interaction beween the particles (fig. 1). The collision of two particles is simulated based on a selectable interaction 


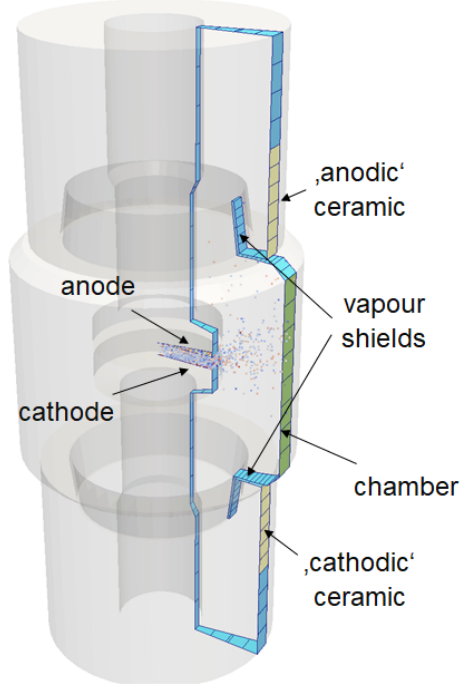

Figure 2. $10^{\circ}$-sector of a simplified VI being simulated in this study with the typical components: contacts formed by the cathode (emits the particles) and the anode, the metallic switching chamber, two vapour shields, and the insulating ceramics.

model. Currently the Variable Hard Sphere model (VHS) [11] and Variable Soft Sphere model (VSS) [14]) are available as both had been taken over from the basic underlying OpenFOAM ${ }^{\circledR}$ DSMC code [15].

\section{Phenomena for different erosion rates/pressure regimes}

Vapour expansion simulations in VIs are helpful for example, when the amount or distribution of the metal deposition on the insulating ceramics for different operation modes and geometries shall be investigated. In the course of such studies we observed that in the low current case the likelihood of particles ejected from the contacts is often higher by factors than that in case of high currents. Also, this likelihood is more sensitive to the wall properties in the low current case. Fig. 2 shows a simplified VI design being used for all simulation results presented in this work and fig. 3 reflects the above mentioned phenomena showing the likelihood for a copper atom to hit the insulator of the VI as function of a constant unipolar current and of the sticking coefficient of the metallic wall chamber.

A possible explanation is that the volume interaction of the metal vapour plays an increasing role with higher erosion rates and surface properties such as the sticking coefficient become more and more unimportant. These assumptions might be quite obvious, but with our previous simulation code there were no means to visualise and quantify the effects. Also when looking to the adsorption of metal vapour on the ceramics it is unclear whether those particles originate directly from wall interactions, e.g. bounce off the stem or the shield surface, or from volume interactions.

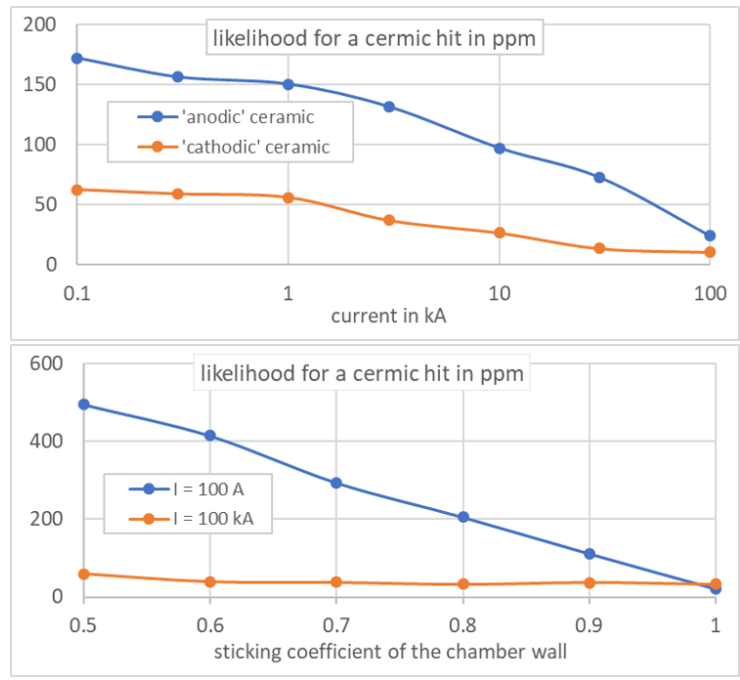

Figure 3. Dependencies of the likelihood for a particle leaving the cathode surface to hit the ceramic insulator on the 'anodic' or 'cathodic' side for the VI geometry as depicted in fig. 2

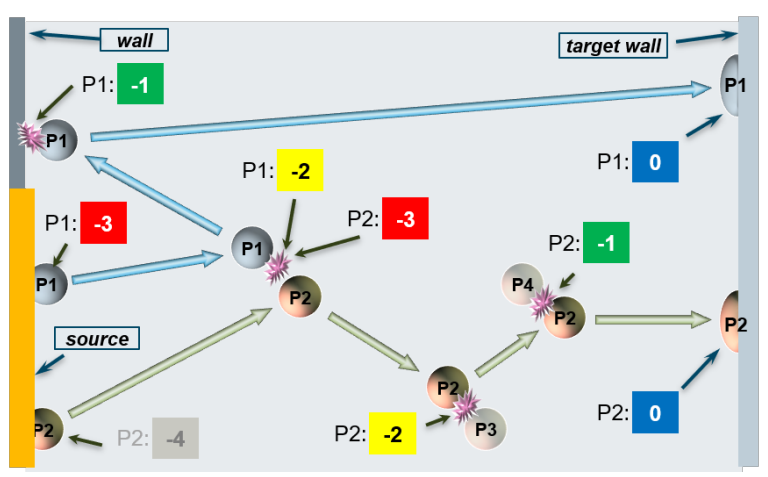

Figure 4. Examples of interaction sequences: Going forward in time, the history for particle P1 is particle creation (c) in the source region (the cathode in all simulations presented in this paper), particle collision $(p)$, wall reflection $(w)$, and finally wall adsorption (a); For particle P2 the total sequence reads c,p,p,p,a. In the following, only the four last interactions (at most) are considered. The indices 0, -1, -2, -3 are described in the text.

\section{Code Extension}

In order to get a better understanding of the mechanisms causing the observed numerical results and to check that these can be explained by physical effects, we extended the existing code by a sort of interaction history functionality. Now various information of each adsorbed particle on a definable target wall are stored and can be visualised. It is possible to specify how many interactions shall be stored for each successful target wall adsorption event. An interaction can be one of the following: (i) particle creation, i.e. the particle is ejected from the particle source, the cathode, (ii) particle-particle interaction, meaning a collision with another particle in the volume, (iii) particle-surface reflection, happening whenever a particle hits a surface, but does not stick to it, (iv) wall adsorption. Together 

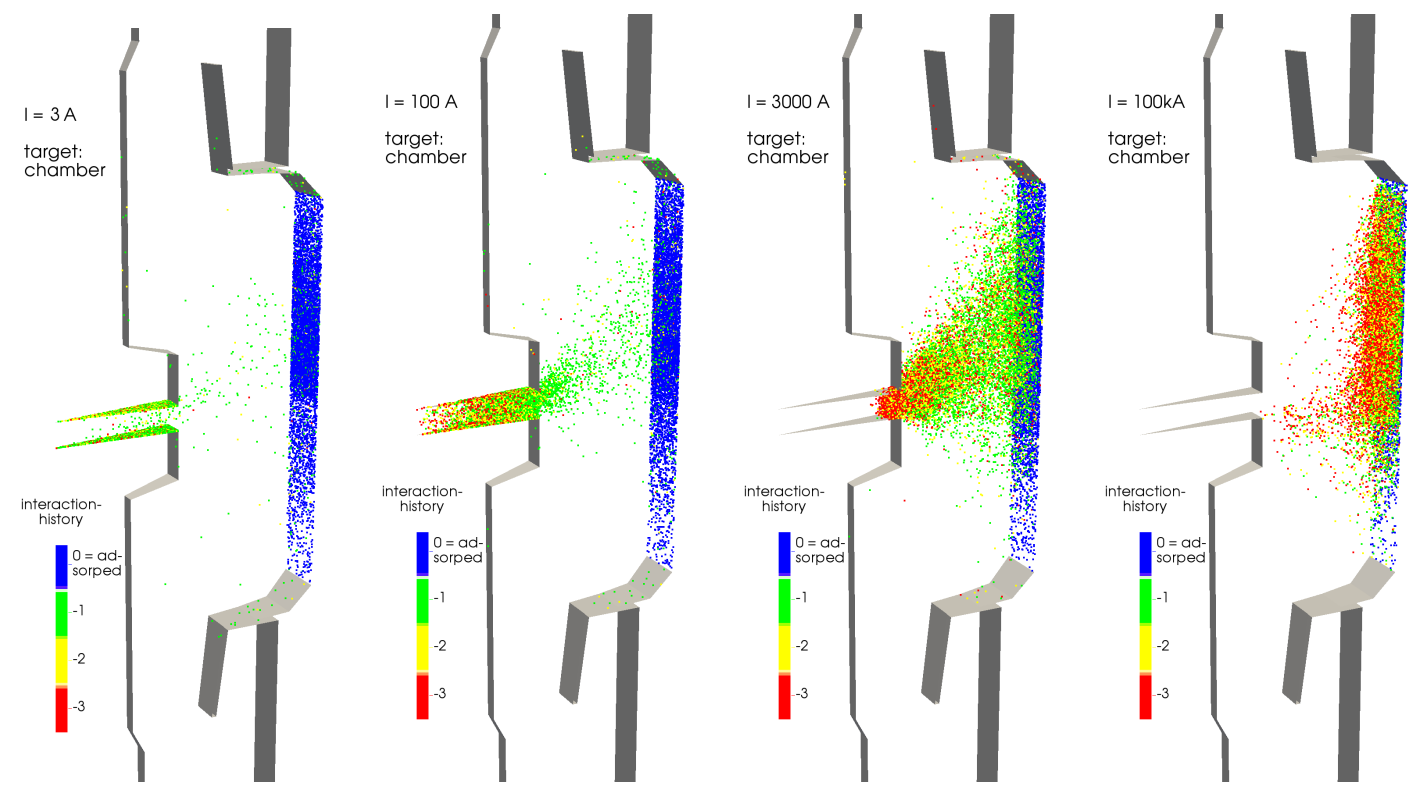

Figure 5. Visualisation of the particle interaction history for all particles ending on the metallic chamber wall after $3 \mathrm{~ms}$ for constant currents of $3 \mathrm{~A}$ (hypothetical), $100 \mathrm{~A}, 3 \mathrm{kA}$ and $100 \mathrm{kA}$.

with the interaction type, the position and the time of the event is recorded. Also the interaction partner (the patch being hit or the particle number in case of a collision) is stored as an additional information. In fig. 4, two exemplary paths with different interaction histories are depicted. In all following figures, interaction sequences are coloured in the same manner; in chronological order: "red" denotes the last but two interaction before sticking (index -3), "yellow" the last but one (-2), and "green" the last interaction (-1) before sticking. The very last interaction (index 0 ) is always the adsorption itself which is colored "blue". From the location one can deduce whether a certain interaction was a particle creation, a wall reflection, or a volume (particle-particle) interaction.

\begin{tabular}{ccccc}
\hline \hline I & DSMCs & \multicolumn{3}{c}{ adsorption on } \\
(kA) & created & anode & chamber & $\begin{array}{c}\text { 'anodic' } \\
\text { ceramic }\end{array}$ \\
\hline 0.1 & 7736327 & 5968957 & 1359772 & 1347 \\
100 & 7786058 & 6129140 & 1344588 & 217 \\
\hline \hline
\end{tabular}

Table 1. Number of DSMC-particles created and adsorbed on various surfaces for $N_{e q}=1 \cdot 10^{7} \cdot I / A$, a setting that generates enough particles hits on the 'anodic' ceramic.

\section{Results and Discussion}

The following simulation results were obtained for the VI design as depicted in fig. 2. The contact radius was $50 \mathrm{~mm}$, the gap between cathode and anode was $10 \mathrm{~mm}$. The chamber inner wall had a radius of $100 \mathrm{~mm}$, the ceramic inner wall radius was $95 \mathrm{~mm}$. The arc is assumed to be a well-behaved diffuse arc burning in an axial magnetic field (AMF) contact.
Thus, in all simulations the cathode was considered to be the sole particle source and it's erosion rate $\dot{m}$ was calculated by $\dot{m}=100 \mu \mathrm{g} / \mathrm{C} \cdot I,[16,17]$ where $I$ is the electric current assumed to be constant for the simulated time of $3 \mathrm{~ms}$ and evenly distributed over the cathode. The created particles are neutral copper atoms only, and the VHS interaction model was applied.

The sticking coefficients were set to 0 for the cathode and 0.8 for the anode. These settings lead to about $78 \%$ of the created particles to get re-adsorbed on the anode (cf. the columns under 'anode' and 'created' in tab. 1 that will be discussed below), and thus about $22 \%$ leaving the gap and being adsorbed on the chamber wall, the 'anodic', and the 'cathodic' ceramic surfaces. The obtained ratio of created and escaping particles appears to be reasonable if one compares it with results reported e.g. in [16]. For all other surfaces the sticking coefficient was set to 0.9 . Only a $10^{\circ}$-sector has been simulated with cyclic boundary conditions on the intersections. These boundary conditions make sure a particle leaving the simulation domain through one of the intersections re-enters the domain on the opposite side at the same radial and axial position with unchanged radial and azimthal velocity components.

As an example of the evaluation of the aforementioned history information, fig. 5 shows the interaction history of those particles which were adsorbed on the chamber wall. From left to the right the particle flux created by the cathode was each time increased by a factor of about 30 starting with a hypothetical value of $3 \mathrm{~A}$ (not reflecting a real copper cathode spot distribution) and ending at $100 \mathrm{kA}$. The corresponding pressures inside the gap were calculated to be $50 \mathrm{mPa}$ for $3 \mathrm{~A}$ to $1.6 \mathrm{kPa}$ for $100 \mathrm{kA}$ at temperatures of about $2500 \mathrm{~K}$. In accordance with [18], less than $25 \%$ of the 


\begin{tabular}{|c|c|c|c|c|c|}
\hline \multirow{3}{*}{$\begin{array}{c}\text { history } \\
\text { index }\end{array}$} & \multirow{3}{*}{$\begin{array}{c}\mathbf{I} \\
(\mathrm{kA})\end{array}$} & \multicolumn{4}{|c|}{ interaction type } \\
\hline & & \multirow[t]{2}{*}{ creation } & \multicolumn{2}{|c|}{ wall } & \multirow[t]{2}{*}{ collision } \\
\hline & & & chamber & other & \\
\hline \multirow{2}{*}{-1} & 0.1 & 0 & 811 & 291 & 245 \\
\hline & 100 & 0 & $\overline{0}$ & $\overline{16}$ & $20 \overline{1}$ \\
\hline \multirow{4}{*}{-2} & 0.1 & 452 & 297 & 139 & 459 \\
\hline & $10 \overline{0}$ & -0 & $\overline{0}$ & $\overline{2} 6$ & $\overline{19} \overline{1}$ \\
\hline & 0.1 & 356 & 123 & 121 & 295 \\
\hline & 100 & 0 & $\overline{0}$ & 18 & $\overline{19} \overline{9}$ \\
\hline
\end{tabular}

Table 2. Details of the interaction history for DSMC particles being adsorbed on the 'anodic' ceramic.

emitted particles are leaving the gap region as most of the particles are adsorbed on the anode. The asymmetry of the particle emission and the deposition on the walls is a result of the fact that only the cathode (the lower contact face) is emitting particles. Note that $N_{\text {eq }}$ was set to $2 \cdot 10^{9} \cdot I / \mathrm{A}$ and is thereby varied linearly with the current (i.e. $N_{\mathrm{eq}}=6 \cdot 10^{9}$ in case of $3 \mathrm{~A}$ and $2 \cdot 10^{14}$ for $100 \mathrm{kA}$ ) leading roughly to $4 \cdot 10^{4}$ created DSMC-particles independent of the actual current in order to obtain similar statistics and numerical noise levels for all runs.

Looking on the green dots in fig. 5, marking the position of the last interaction before the DSMC-particle hits the chamber, we observe the expected. For an extremely low and hypothetical current, the particles originating from the cathode would directly hit the chamber more or less without any further interaction. This corresponds to a surface to surface radiation without any volume scattering or absorption. At $I=100 \mathrm{~A}$, the virtual source of the particles in this geometry is the transition area from the gap between the contacts and the chamber volume. Starting from there, hardly any further volume interaction takes place. Increasing the current further, the number of volume interactions increases, too. And finally at $I=100 \mathrm{kA}$, there is not a single particle that originates directly from the cathode, the primary particle source.

Performing a similar analysis, but this time with respect to the 'anodic' ceramic, we get the results tabulated in tab. 1 and tab. 2 (low and high current cases) and shown in fig. 6 (low current case). $N_{\text {eq }}$ was set to $10^{7} \cdot I / \mathrm{A}$, so again the number of tracked DSMCparticles is the same for both currents, but this time a factor 200 higher. This is needed in order to get a reasonable number of particles on the ceramics. Tab. 1 lists more general numbers with respect to the different hit rates: At $0.1 \mathrm{kA}$ the likelihood to hit the anodic ceramic for a particle leaving the cathode is a factor of 6 higher than in case of $100 \mathrm{kA}$. However, since $N_{\text {eq }}(100 \mathrm{kA})$ is a factor of $10^{3}$ higher than $N_{\text {eq }}(0.1 \mathrm{kA})$, in absolute terms the deposition produced per unit time is a factor of 160 higher in the high current case.

From tab. 2 one can see that for both current levels
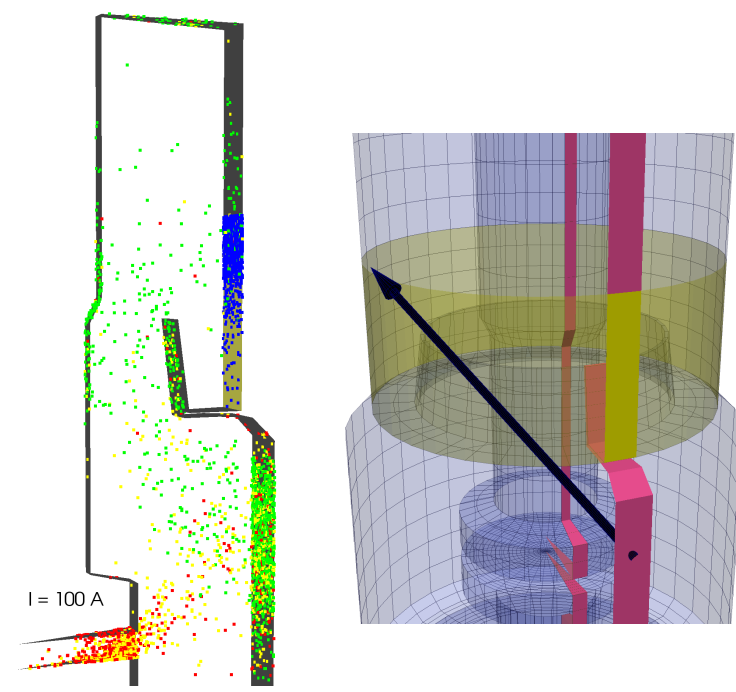

Figure 6. Left: The particle interaction history for all particles sticking to the 'anodic' ceramic wall after $3 \mathrm{~ms}$ for a constant current of $100 \mathrm{~A}$. Right: The arrow shows the direct line of sight between contact stem and shield from the chamber wall to the ceramic.

no particle from the contact surface hits the ceramic directly as we have zero particles created for the history index ' -1 '. This of course is a quite general goal in VI design, namely, to avoid direct sputtering of the insulator by the primary particle source. In case of a high evaporation rate, however, it takes more than three interactions for a particle originating from the chamber wall to reach the 'anodic' ceramic (0 particles for index -3 under 'chamber'), which accommodates for the fact of a large pressure of the order of $\mathrm{Pa}$ in that region, corresponding to a mean free path of the order of millimetres. The main source of particles hitting the examined ceramic in this arrangement are volume (collision) interactions. In contrast to this, tracing back two interactions in the low current case, one finds 452 particles that are created, meaning one third of all particles being adsorbed on the 'anodic' ceramic (1347) do have a single interaction after being emitted from the cathode: most likely this is the reflection at the chamber wall, explaining the influence of the impact of sticking coefficient here. This is supported by the fact that 811 particles of 1347 originate directly from the chamber.

As previously assumed for the low current case $(100 \mathrm{~A})$, the main source of the particles hitting the ceramic is the switching chamber which appears greenish in the left of fig. 6, indicating that this was the last interaction before adsorption. The vast majority of particles has gone through the vacuum without any further volume interaction. This is possible since there exists a direct line of sight from the chamber wall to the ceramic as shown on the right of fig. 6 . This fact might not be evident when looking on the cross section of the VI only. However, one has to bear in mind that whenever a particle leaves the calculation domain via one of the intersection faces, the cartesian 
velocity components change as a result of the $10^{\circ}$ angle between the intersection surface normals. As a consequence, when tracking a non interacting particle moving on a straight line, it appears to be bend radially outward each time it crosses the intersection. Hence, this particle apparently moves on a curved line reaching areas that seem to be occluded in the $2 \mathrm{D}$ cross section.

\section{Conclusions}

The results are obtained for a particular VI geometry with AMF contacts assuming the cathode surface as vapour source region. They document that at high currents volume interaction (particle-particle collisions) plays the most important role and wall interactions (reflection and desorption by inner surfaces) are less important. At low currents, on the contrary, the most likely sputter mechanism is the reflection of the particles at the metallic VI chamber wall. Volume interaction and reflection by other surfaces are less important for the VI geometry in this case.

The new feature of our DSMC code improves the understanding of metal vapour expansion and deposition in a VI stressed by operational and short circuit currents, and it helps to identify critical issues occurring in the course of VI development. However, up to now, the simulation is not accurate by its own, but rather needs to be compared and calibrated with experiments. Care should be taken to interpret the simulation of real switching operations, because the erosion rate might be different and the source region can be non-uniform, particularly if the VI is close to its breaking limit and the anode takes over an active particle emitter role.

\section{References}

[1] P. Slade. The Vacuum Interrupter: Theory, Design, and Application. 1st Edition. CRC Press, Taylor and Francis Group, London, 2008.

[2] H. Fink, D. Gentsch, and M. Heimbach. Condensed metal vapor on alumina ceramic in vacuum interrupters. IEEE Trans. Dielectr. Electr. Insul., 9(2), 2002. doi:10.1109/94.993736.

[3] B. Kuehn, M. Kurrat, M. Hilbert, I. Gramberg, and D. Gentsch. Characterization of metal vapor deposition on vacuum interrupter ceramics and its impact on electric field distribution. IEEE Trans. Dielectr. Electr. Insul., 24(6), 2017. doi:10.1109/TDEI.2017.006486.

[4] H. Schellekens and J. Battandier. Metal vapor deposition on ceramic insulators and vacuum interrupter lifetime prediction. In Proc. 19th Inter'nl Symp. on Disch. and Electr. Insulation in Vac., volume 1, 2000. doi:10.1109/DEIV.2000.877270.

[5] I. S. Gramberg, M. Kurrat, and D. Gentsch. Investigations of copper chrome coatings on vacuum circuit breaker ceramics by electron probe microanalysis and electric field simulation. IEEE Transactions on Plasma Science, 41, 2013.

doi:10.1109/TPS.2013.2273260.
[6] B. Kuehn, M. Kurrat, M. Hilbert, and D. Gentsch. Multiple shield arrangements breakdown model in vacuum interrupters. In 201627 th International Symposium on Discharges and Electrical Insulation in Vacuum (ISDEIV), volume 2, pages 1-4, 2016. doi:10.1109/DEIV.2016.7763948.

[7] M. Kurrat. Vacuum arc ion flux from vacuum interrupter contact gap. Plasma Physics and Technology Journal, 4, 2017. doi:10.14311/ppt.2017.2.186.

[8] E. D. Taylor. Application of research in field emitter arrays to the breakdown of contacts in vacuum. In 2012 25th International Symposium on Discharges and Electrical Insulation in Vacuum (ISDEIV), pages 41-44, 2012. doi:10.1109/DEIV.2012.6412445.

[9] G. Bird. Approach to translational equilibrium in a rigid sphere gas. Physics of Fluids, 6, 1963. doi:10.1063/1.1710976.

[10] A. Geisler and N. Wenzel. Metal vapor deposition in vacuum interrupters: Utilization of dsmc methods and considerations on vapor sources. 28th International Symposium on Discharges and Electrical Insulation in Vacuum, 2, 2018. doi:10.1109/DEIV.2018.8537030.

[11] G. Bird. Molecular Gas Dynamics and the Direct Simulation of Gas Flows. Number Bd. 1 in Molecular Gas Dynamics and the Direct Simulation of Gas Flows. Clarendon Press, 1994.

[12] T. Scanlon, E. Roohi, C. White, M. Darbandi, and J. Reese. An open source, parallel dsmc code for rarefied gas flows in arbitrary geometries. Computers and Fluids, 39, 2010. doi:10.1016/j. compfluid.2010.07.014.

[13] K. Hencken. Investigation of metallic vapor condensation in a vacuum interrupter using dsmcfoam. 9th OpenFOAM ${ }^{\circledR}$ Workshop, Zagrep, Croatia, 2014.

[14] K. Koura and H. Matsumoto. Variable soft sphere molecular model for air species. Physics of Fluids A: Fluid Dynamics, 4(5), 1992. doi:10.1063/1.858262.

[15] H. G. Weller, G. Tabor, H. Jasak, and C. Fureby. A tensorial approach to computational continuum mechanics using object-oriented techniques. Computers in Physics, 12(6), 1998. doi:10.1063/1.168744.

[16] G. Farrall, F. Hudda, and J. Toney. The time-resolved characterization of erosion products from high-current, copper vacuum arcs. Plasma Science, IEEE Transactions on, 11, 1983. doi:10.1109/TPS.1983.4316240.

[17] J. Kutzner, R. Batura, and Z. Stachowiak. Experimental analysis of the electrode products emitted by high-current vacuum arcs. IEEE Trans. Plasma Sci., 27(4), 1999. doi:10.1109/27.782255.

[18] M. B. Schulman, P. G. Slade, and J. A. Bindas. Effective erosion rates for selected contact materials in low-voltage contactors. IEEE Transactions on Components, Packaging, and Manufacturing Technology: Part A, 18(2), 1995. doi:10.1109/95.390312. 